\title{
Doping of epitaxial graphene by direct incorporation of nickel adatoms
}

Received 1st February 2019, Accepted 00th Xxxxxxx 2019

DOI: $10.1039 / x 0 x x 00000 x$

\author{
Virginia Carnevali, ${ }^{1}$ Laerte L. Patera ${ }^{1,2,+}$ Gianluca Prandini, ${ }^{1,{ }^{\ddagger}}$ Matteo Jugovac, ${ }^{1, \#}$ Silvio Modesti, ${ }^{1,2}$ \\ Giovanni Comelli, ${ }^{1,2}$ Maria Peressi, ${ }^{1,3, *}$ and Cristina Africh ${ }^{2, *}$
}

\begin{abstract}
Direct incorporation of $\mathrm{Ni}$ adatoms during graphene growth on $\mathrm{Ni}(111)$ is evidenced by Scanning Tunneling Microscopy. The structure and energetics of the observed defects is thoroughly characterized at the atomic level on the basis of density functional theory calculations. Our results show the feasibility of a simple scalable method, which could be potentially used for the realization of macroscopic practical devices, to dope graphene with a transition metal. The method exploits the kinetics of the growth process for the incorporation of Ni adatoms in the graphene network.
\end{abstract}

\section{Introduction}

Future applications of graphene-based devices rely on the capability of tailoring its electronic, magnetic and chemical properties in a controllable way. For a similar aim, defectengineering is a widely exploited approach in the semiconductor industry, allowing control over the carrier type and density. Following this strategy, recent studies revealed the actual possibility of introducing doping defects in graphene to tune its properties. ${ }^{1-3}$ For example, by adding proper gaseous precursor during the Chemical Vapor Deposition (CVD) process ${ }^{4}$ or by means of low-energy ion implantation, ${ }^{5,6}$ substitutional nitrogen atoms can be trapped inside carbon vacancies, strongly modifying the graphene electronic structure. $^{7}$ New functionalities are predicted to arise when transition metal dopants are introduced. In particular, it is possible to induce a magnetic moment ${ }^{8}$ and to add chemical

${ }^{1}$ Università di Trieste - Dipartimento di Fisica, via Valerio 2, Trieste, Italy

2 IOM-CNR Laboratorio TASC, S.S. 14 km 163.5 in AREA Science Park, Basovizza, I34149 Trieste, Italy

3 IOM-CNR DEMOCRITOS National Simulation Center, via Bonomea 265, 1-34136 Trieste, Italy

${ }^{+}$Present address: Institute of Experimental and Applied Physics, University of Regensburg, D-93053 Regensburg, Germany

Present address: École Polytechnique Fédérale de Lausanne, CH-1015 Lausanne, Switzerland

" Present address: Peter Grünberg Institute (PGI-6), Forschungszentrum Jülich, 52425 Jülich, Germany

Corresponding Authors: peressi@ts.infn.it (theory), africh@iom.cnr.it (experiments)

Electronic Supplementary Information (ESI) available: STM images showing: the decrease of the $\mathrm{Ni}$-doping defects concentration when increasing the growing temperature; the appearance of the defects by varying the tunneling current and the bias; different equivalent orientations of a selected $\mathrm{Ni}$-doping defect. STS data showing the differential conductance in the Fermi level region and a possible

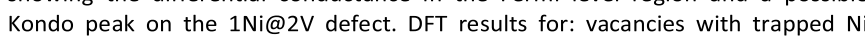
adatoms (additional simulated STM images with the sketch of the $\mathrm{C}$ atoms delimiting the defects); simulated STM images of the 1Ni@3V(2top+1fcc) defect at different bias; empty vacancies in epitaxial graphene (simulated STM images, formation and binding energies); atomic projected density of states for the 1Ni@2V and 1Ni@3V defects. See DOI: 10.1039/x0xx00000x activity, $^{9,10}$ enhancing the catalytic behavior of the layer with respect to small gaseous molecules of environmental importance. ${ }^{11}$ For example, as shown by Qiu et al., single Ni atoms trapped in graphene exhibit an unusual catalytic performance in the hydrogen evolution reaction. ${ }^{12}$ However, despite these appealing potential properties, only few experimental realizations have been achieved so $\mathrm{far}^{12-16}$ and an approach to obtain metal-doped graphene in a single growth step has not yet been reported. In principle, a single step process usable for the preparation of macroscopic graphene flakes, without requiring the preliminary formation of graphene vacancies, would be simpler and more likely to offer the possibility of fine controlling the doping level of the resulting layer.

In this work, we show that graphene doping can be obtained by direct incorporation of individual nickel ( $\mathrm{Ni}$ ) atoms during the formation of epitaxial graphene layers by CVD on a $\mathrm{Ni}(111)$ surface. The atomic configuration of different defects was determined by a combination of high-resolution scanning tunneling microscopy (STM) and density-functional theory (DFT) calculations. The stability and the bonding configuration of the observed $\mathrm{Ni}$-doping defects are discussed in light of the calculated charge distribution.

\section{Results and discussion}

A typical STM image of an epitaxial graphene (EG) layer grown on $\mathrm{Ni}(111)$ at $400^{\circ} \mathrm{C}$, using ethylene as carbon source, ${ }^{17}$ is shown in Figure 1. Under these conditions, surface carbide, if present, readily converts to graphene and it is possible to obtain extended graphene domains (up to few hundreds of $\mathrm{nm}$ wide) ${ }^{18}$ with a low concentration of domain boundaries. ${ }^{19}$ However, at this low growth temperature, the layer is not perfect: a sizable number (about $1 \%$ of graphene $\mathrm{C}$ atoms) of point-like bright defects are created, both as isolated features and short chain structures. The formation of these defects is independent of the growth mechanism (via carbide 


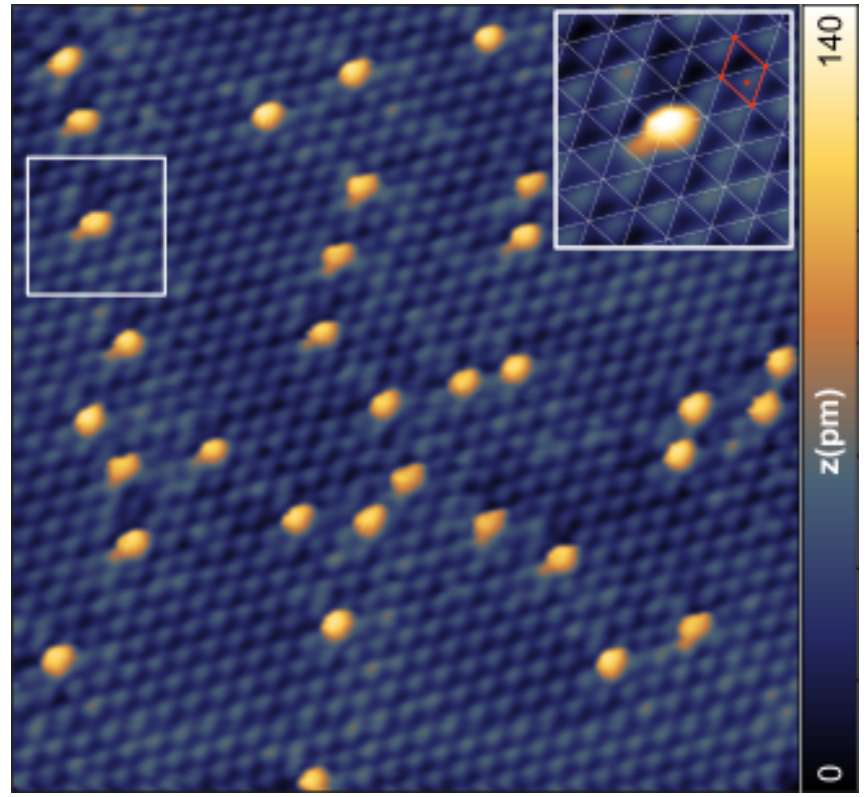

Figure 1. Defective epitaxial graphene on $\mathrm{Ni}(111)$. Inset: zoom-in on a defect structure. The white grid intersects on top sites; a primitive unit cell of the $\mathrm{Ni}(111)$ surface is marked in red, with the position of the carbon atoms of the epitaxial graphene layer indicated by dots. The vertical scale bar shows the apparent height. Image size: $8 \times 8$ $\mathrm{nm}^{2} ; \mathrm{V}_{\text {bias }}=0.1 \mathrm{~V}, \mathrm{I}=2 \mathrm{nA}$.

conversion or direct graphene growth); once formed, these defects appear to be stable up to graphene dissolution temperatures. The exact concentration of these bright defects depends on the growth temperature, with larger defect densities obtained at lower temperatures $\left(350-400^{\circ} \mathrm{C}\right)$ and no point-defects detected above $600^{\circ} \mathrm{C}$ (see Figure S1 in ESI). Their apparent height ranges from about 0.5 to $1.5 \AA$, strongly depending on the tunneling current, i.e. from the tip-sample distance (see Figure S2 in ESI).

The appearance of these bright objects is clearly different from that of the single carbon vacancies and Stone-Wales (SW) defects previously reported for CVD graphene grown on Ni(111). ${ }^{20}$ Based on their width and height, and on the well-known presence of mobile adatoms on metal surfaces at these temperatures, we previously tentatively assigned the point-like defects to substitutional $\mathrm{Ni}$ atoms trapped in the carbon network during the growth process. ${ }^{17}$ This identification was also in line with previous first-principles calculations, indicating that the formation energy of vacancy structures is greatly reduced compared to free-standing graphene when the dangling bonds are passivated either by metal surface atoms or adatoms. ${ }^{21}$ More recently, by combining direct STM imaging at the millisecond time scale with DFT and molecular dynamics simulations, we clearly identified individual $\mathrm{Ni}$ adatoms catalyzing the graphene growth on the Ni substrate, by temporarily attaching at kink sites along the edges of growing graphene flakes. ${ }^{22}$ Figure 2 shows selected frames of a high-speed movie (frame rate $60 \mathrm{~Hz}$ ) acquired during graphene growth at $440{ }^{\circ} \mathrm{C}$ : the synthesis proceeds with the same mechanisms elucidated in our previous work but in this case two $\mathrm{Ni}$ atoms remain trapped into the network. In more details, starting from an atomically straight Klein (k) edge (at $0 \mathrm{~ms}$ ), after $33 \mathrm{~ms}$ a kink site can be observed in the middle of the image, as well as a $\mathrm{Ni}$ adatom (imaged as a bright protrusion), catalyzing the $\mathrm{C}$ addition process. ${ }^{22}$ Detachment of the $\mathrm{Ni}$ adatom from the kink site $(66 \mathrm{~ms})$ interrupts the growth along the edge. A counter-propagating growing front, carrying an opposite kink, comes from the top of the frame, thanks to the action of another $\mathrm{Ni}$ adatom, which gets stably trapped (132 ms) against the preexisting kink, due to the higher coordination. As the growth progresses $(19,156 \mathrm{~ms})$, a second $\mathrm{Ni}$ adatom is stably incorporated close to the first one in the carbon network (see below). This definitely proves that the observed point-like bright defects are Ni doping centers in the graphene layer.

A closer inspection of Figure 1 allows distinguishing various configurations of Ni-dopants, characterized by different appearances. Our high-resolution STM images indicate a top-fcc registry of graphene with respect to the $\mathrm{Ni}(111)$ surface, ${ }^{19}$ which in turn allows the position and the orientation of different $\mathrm{Ni}$ doping configurations to be identified with respect to the graphene lattice, as shown for one of the observed defects in the zoomed region of the figure. On the basis of the experimental findings, we simulated, using a density functional theory approach, several $\mathrm{Ni}$ doping configurations, characterized by a variable number of missing carbon atoms (from 1 to 5) and containing 1 or 2 trapped $\mathrm{Ni}$ adatoms (Figure 3; see also Figures S3 and S4 in ESI). The dangling bonds in the graphene network are passivated by the substrate and by the trapped $\mathrm{Ni}$ adatom(s), which leads in all cases to a localized bright feature in simulated STM images. We classify the observed defects according to the number $(n)$ and position (top or hollow-fcc, hereafter shortly indicated as $f c c$ ) with respect to the Ni lattice of the carbon vacancies $(V)$ and the number $(m)$ of the Ni-doping atoms. Thus, for example, 2Ni@4V(2top+2fcc) indicates a defect with $2 \mathrm{Ni}$-doping atoms trapped in a cluster of 4 vacancies, where 2 top and 2 hollow-fcc carbon atoms are missing. The remarkable agreement between the simulated (middle panel) and experimental (bottom panel) STM images allows safe identification of the main $\mathrm{Ni}$ doping configurations observed in our measurements with the optimized atomic structures obtained by DFT (upper panel). The different defect configurations described above always coexist in the layer, in concentrations that vary with the growth parameters. However, 1Ni@2V, 1Ni@3V(1top+2fcc) and, to a lesser extent,

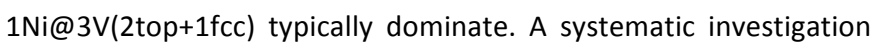
of the influence of each growth parameter was beyond the scope of the present work.

For the1 $1 \mathrm{Ni@1V(1top)} \mathrm{and} \mathrm{1Ni@2V(1top+1fcc)} \mathrm{configurations,} \mathrm{DFT}$ calculations show that the dangling bonds (DBs) left behind by the missing $\mathrm{C}$ atom(s) are all passivated by the trapped $\mathrm{Ni}$ adatom. For the $1 \mathrm{Ni} @ 2 \mathrm{~V}(1 \mathrm{top}+1 \mathrm{fcc})$ case, this implies that the shape of the $\mathrm{Ni}$ adatom appears asymmetric, with two protrusions towards the closest hollow-fcc $\mathrm{C}$ atoms at the defect edge. Due to the symmetry 

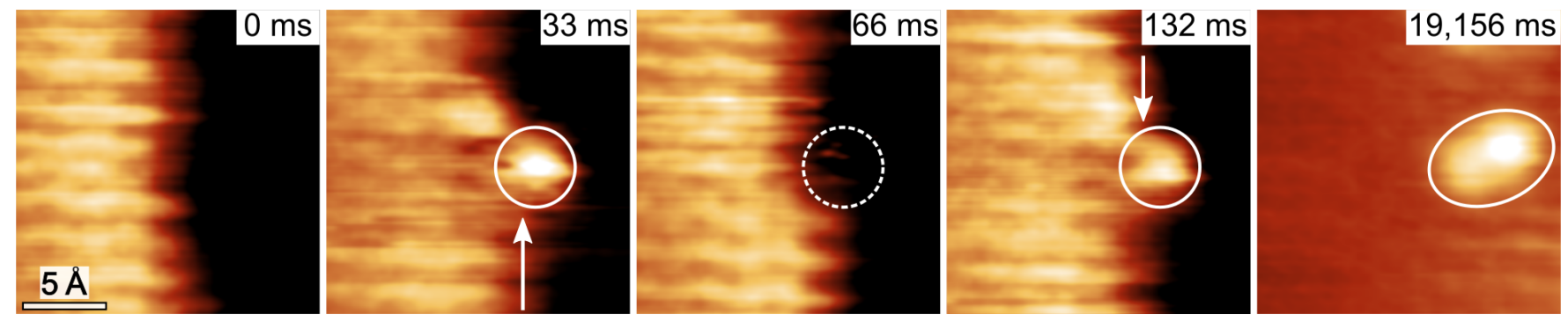

Figure 2: Selected frames of a high-speed STM sequence acquired at $440{ }^{\circ} \mathrm{C}$ in quasi-constant height mode at the $\mathrm{k}$ edge of a growing graphene flake. White arrows and circles indicate the position of the kink sites along the growing direction of the graphene edge and of the $\mathrm{Ni}$ adatom $(\mathrm{s})$, respectively $\left[\mathrm{V}_{\text {bias }}=0.02 \mathrm{~V} ; \mathrm{I}=7 \mathrm{nA}\right.$; frame rate $=60 \mathrm{~Hz}]$.

of this defect, three equivalent orientations, rotated by $120^{\circ}$, are expected and indeed experimentally observed (Figure S3 in ESI).

The $\mathrm{Ni}$ adatom sits in the graphene plane for all the observed defects, with the exception of 1Ni@1V(1 top), where, due to steric effect, it is placed at about $0.9 \AA$ above the carbon layer. This result is at slight variance with a previous report for EG on $\mathrm{Cu}(111)$, where the $\mathrm{Cu}$ adatom in comparable defects lies in between the surface and the graphene plane. ${ }^{21}$ The different equilibrium configuration of the embedded adatoms can be rationalized in terms of the different structural matching of EG with $\mathrm{Cu}(111)$ vs $\mathrm{Ni}(111)$ on the one side, and of the different strength of the $\mathrm{C}-\mathrm{Cu}$ vs $\mathrm{C}-\mathrm{Ni}$ interaction on the other.

An asymmetry in the appearance of the defect characterizes also the $3 \mathrm{~V}$ s. Here a single embedded $\mathrm{Ni}$ adatom cannot saturate all the $D B s$, yet there is not enough space for two $\mathrm{Ni}$ atoms. The remaining DBs are thus passivated by the substrate, which for 1Ni@3V(2 top + $1 \mathrm{fcc}$ ) leads to a surface $\mathrm{Ni}$ atom partially lifted above the outermost metallic plane.

We calculated the formation energy of the $m \mathrm{Ni} @ n V$ defects with respect to a perfect epitaxial graphene layer and the reservoir of $\mathrm{C}$ atoms in graphene and $\mathrm{Ni}$ atoms in the bulk: ${ }^{21}$

$E_{\text {form }}=E_{\text {def } G / N i}+n E_{C G}-E_{G / N i}-m E_{N i b u l k}$

where $E_{\text {def } G / N i}$ is the total energy of the defective system ( $\mathrm{Ni}(111)$ substrate + defected graphene with possible trapped adatoms), $E_{G / N i}$ is the total energy of the corresponding perfect system (pristine graphene on $\mathrm{Ni}(111)$ substrate, with the same dimensions of the defected one), $E_{C G}$ is the total energy of one $C$ atom in the free standing graphene, and $E_{\mathrm{Ni} \text { bulk }}$ is the total energy of a $\mathrm{Ni}$ atom in bulk. Using this definition, the defect formation energy is positive; the larger its value, the higher its formation cost in the otherwise perfect system. Resulting energy values for the observed defects are reported below the stick-and-ball models in Figure 3. Our values are in line with those obtained by Wang et al. for comparable defects. ${ }^{21}$ The higher the number of carbon vacancies, the higher the defect formation energy.

It has to be noted that the energetics of the different configurations cannot be directly linked to the abundance of the formed defects. Indeed, the energies reported here refer to the removal of $\mathrm{C}$ atoms from a perfect graphene layer accompanied by the addition of $\mathrm{Ni}$ atoms in the vacancies. These energies are all very high, implying that Ni-doping starting from a perfect graphene layer is extremely difficult to obtain in real systems. Production of a Ni-doped graphene layer becomes much easier by introducing $\mathrm{Ni}$-doping atoms directly during the graphene growth, exploiting the kinetics of the growth process. In turn, this also affects the relative abundance of the different defect configurations.

For the sake of completeness, we simulated also the structure and appearance of empty graphene vacancies. In particular, we investigated $1 \mathrm{~V}(1$ top), $2 \mathrm{~V}$, and $3 \mathrm{~V}(2 \mathrm{top}+1 \mathrm{fcc})$, corresponding to the three leftmost defects in Figure 3 without the $\mathrm{Ni}$ adatoms (see Figure S6 in ESI). Remarkably, in all cases the simulated images of the bare defects (without $\mathrm{Ni}$ adatoms inside) do not show a bright feature but rather a dark appearance. Most of the terminal carbon atoms in fcc sites delimiting the defects are bent toward the metal, as already reported for substrate passivated graphene edges. ${ }^{23}$ Furthermore, the simulations rule out the possibility that the $\mathrm{Ni}$ doping adatoms are substrate atoms lifted by the defect: the surface $\mathrm{Ni}$ atom underneath is slightly lifted with respect to the surface but not enough to lie in the graphene plane, thus the appearance of the empty vacancy remains dark. Since in the STM images of our samples we only very rarely observe defects with dark appearance, the results of our simulations strongly support the presence of $\mathrm{Ni}$-doping adatoms in the defects we describe.

Although in our calculations we do not mimic the dynamics of the adatom trapping process, nor have access to realistic barrier formation, we can make reference to the empty vacancies in order to estimate the stability of the trapped $\mathrm{Ni}$ adatom. Focusing on the defects including only one $\mathrm{Ni}$ adatom, which are the most common, we subtract, from the total energy of the defective structure as a whole (vacancy with the adatom, $\left.E_{\text {def G/Ni }}(1 \mathrm{Ni} @ \mathrm{nV})\right)$, the sum of the total energies of the empty vacancy passivated by the substrate $E_{d e f}$ $G / N i(n V)$ and of an isolated $\mathrm{Ni}$ atom $\left(E_{N i \text { is }}\right)$. The energy difference is always negative, indicating a strong binding of the adatom with the vacancy. More specifically, we obtain $-4.08 \mathrm{eV},-5.61 \mathrm{eV}$ and -5.72 eV for1Ni@1V(1top), 1Ni@2V(1top+1fcc), and 1Ni@3V(2top+1fcc), respectively. These values represent the energy gained by an optimized passivation of the DBs at the graphene vacancy by the trapped $\mathrm{Ni}$ adatom. They are comparable, in absolute value, with the cohesion energy of $\mathrm{Ni}$ bulk $(4.87 \mathrm{eV} /$ atom calculated in this work), and therefore stronger than the binding energy of a $\mathrm{Ni}$ adatom on the clean $\mathrm{Ni}(111)$ surface.

Electron density difference plots provide further indications of the binding of the adatom with the vacancy. The electron density 


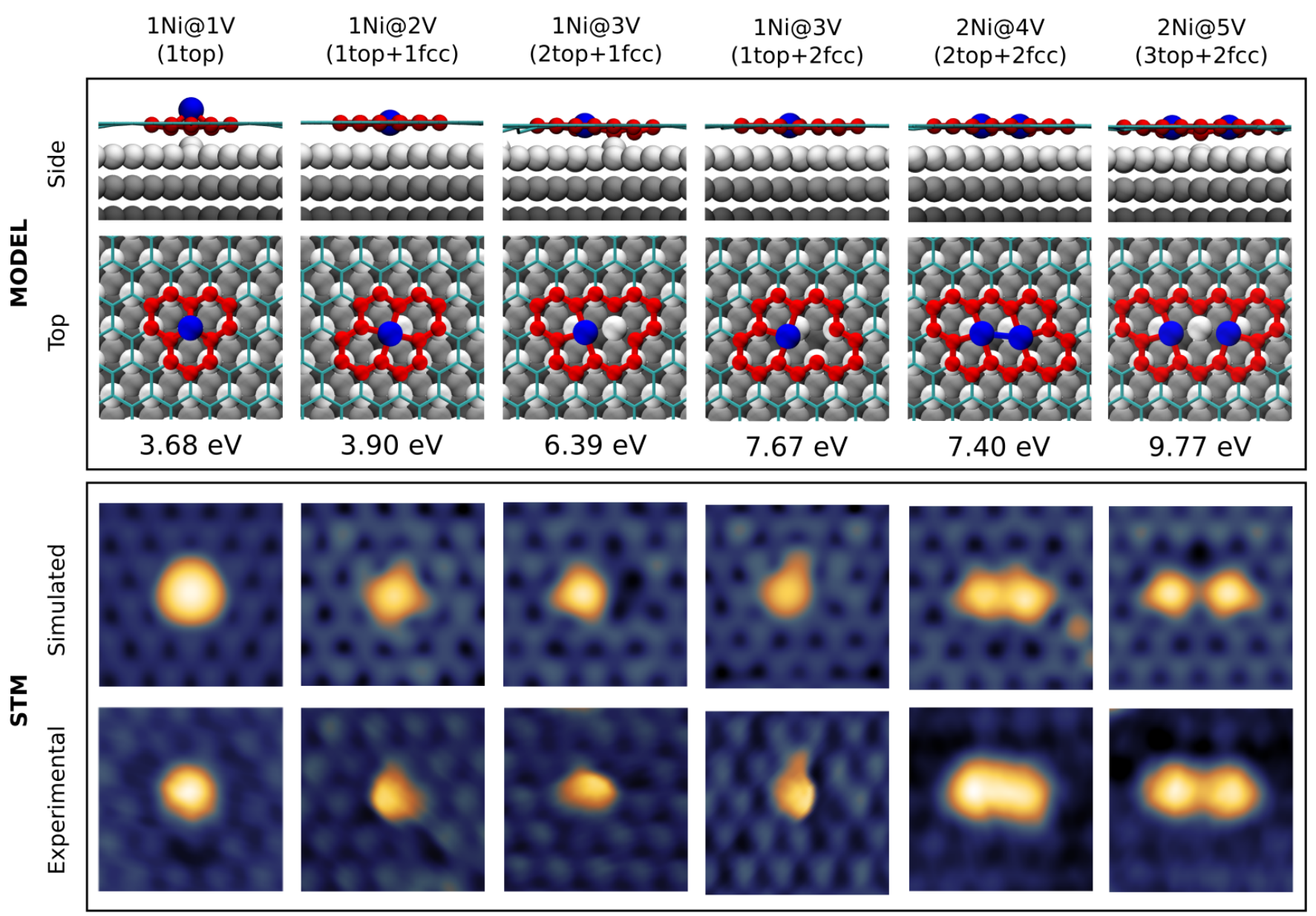

Figure 3: Ni dopant configurations in graphene on $\mathrm{Ni}(111)$. Top panel: stick-and-ball model of DFT relaxed structures. Formation energies are indicated below each structure. Bottom panels: DFT simulated and experimental STM images. Image size: $1.2 \times 1.2 \mathrm{~nm}^{2}$. The different structures are classified depending on the number of embedded $\mathrm{Ni}$ adatoms, and on the number and position of $\mathrm{C}$ vacancies $(\mathrm{V})$. Computational parameters: $\mathrm{V}_{\text {bias }}=-0.3 \mathrm{~V}$; ILDOS isosurface lying $\approx 2 \AA$ above graphene and with ILDOS value of $5 \cdot 10^{-}$ ${ }^{5}|\mathrm{e}| / \mathrm{a}_{0}{ }^{3}$. Experimental parameters: $1 \mathrm{Ni} @ 1 \mathrm{~V}(1 \mathrm{top})\left[\mathrm{V}_{\text {bias }}=-0.1 \mathrm{~V} ; \mathrm{I}=\right.$ $1 \mathrm{nA}], 1 \mathrm{Ni} @ 2 \mathrm{~V}(1 \mathrm{top}+1 \mathrm{fcc})\left[\mathrm{V}_{\text {bias }}=-0.1 \mathrm{~V} ; \mathrm{I}=1 \mathrm{nA}\right], 1 \mathrm{Ni} @ 3 \mathrm{~V}$ $(2 \mathrm{top}+1 \mathrm{fcc})\left[\mathrm{V}_{\text {bias }}=-0.3 \mathrm{~V} ; \mathrm{I}=2 \mathrm{nA}\right], 1 \mathrm{Ni} @ 3 \mathrm{~V}(1 \mathrm{top}+2 \mathrm{fcc})\left[\mathrm{V}_{\text {bias }}=-\right.$ $0.3 \mathrm{~V} ; \mathrm{I}=2 \mathrm{nA}], 2 \mathrm{Ni} @ 4 \mathrm{~V}(2 \mathrm{top}+2 \mathrm{fcc})\left[\mathrm{V}_{\text {bias }}=-0.3 \mathrm{~V} ; \mathrm{I}=1 \mathrm{nA}\right]$ and $2 \mathrm{Ni} @ 5 \mathrm{~V}(3 \mathrm{top}+2 \mathrm{fcc})\left[\mathrm{V}_{\text {bias }}=-0.3 \mathrm{~V} ; \mathrm{I}=1 \mathrm{nA}\right]$.

difference is calculated by subtracting from the electron density distribution of the whole defective structure, the one of the structure with the empty vacancy and the one of the trapped adatom, both kept in the frozen geometry that they have in the defective structure filled by $\mathrm{Ni}$. The plots, reported in Figure 4, show that the most pronounced electron density rearrangement occurs between the trapped $\mathrm{Ni}$ atom and the borders of the graphene vacancy rather than with the substrate. Indeed, if we increase the isosurface value, the modified charge regions between the $\mathrm{Ni}$ adatom and the substrate vanish, while they are still present at the borders of the vacancy. The electron density distribution thus indicates that the $\mathrm{Ni}$ adatom is more strongly bound to graphene than to the substrate, suggesting the remarkable possibility of maintaining the $\mathrm{Ni}$-doping even after decoupling the graphene layer from the Ni substrate, to transfer it elsewhere.

As a final point, we briefly address the electronic and magnetic properties of the $\mathrm{Ni}$-doping centers. DFT simulations indicate that the single trapped $\mathrm{Ni}$ adatom carries a small magnetic moment $(\mu=$ 0.03-0.12 $\mu_{\mathrm{B}}$, see Figure $\mathrm{S} 7$ in ESI), similarly to the findings reported for a $\mathrm{Ni}$ atom embedded in $1 \mathrm{~V}$ and $2 \mathrm{~V}$ in free-standing graphene. ${ }^{8}$ Differential conductance ( $\mathrm{dl} / \mathrm{dV}$ ) measurements performed at $4.2 \mathrm{~K}$ with a commercial Omicron LT-STM show a marked zero-bias peak (ZBP) only for the 1Ni@2V defects (see Figure S8 in ESI). As discussed in the ESI, such feature can be potentially related to a Kondo resonance.

\section{Experimental and computational methods}

The experiments were performed in a UHV system (base pressure $=$ $1 \cdot 10^{-10} \mathrm{mbar}$ ) equipped with standard sample preparation facilities and with an Omicron variable temperature STM. After several cycles of $\mathrm{Ar}^{+}$sputtering and annealing $\left(600^{\circ} \mathrm{C}\right)$, epitaxial graphene was prepared on $\mathrm{Ni}(111)$ by ethylene exposure $\left(\mathrm{p}=1 \cdot 10^{-7} \mathrm{mbar}\right)$, or $\mathrm{C}$ segregation from the bulk, between 350 and $600^{\circ} \mathrm{C}$. Imaging was performed in constant-current mode at room temperature. 

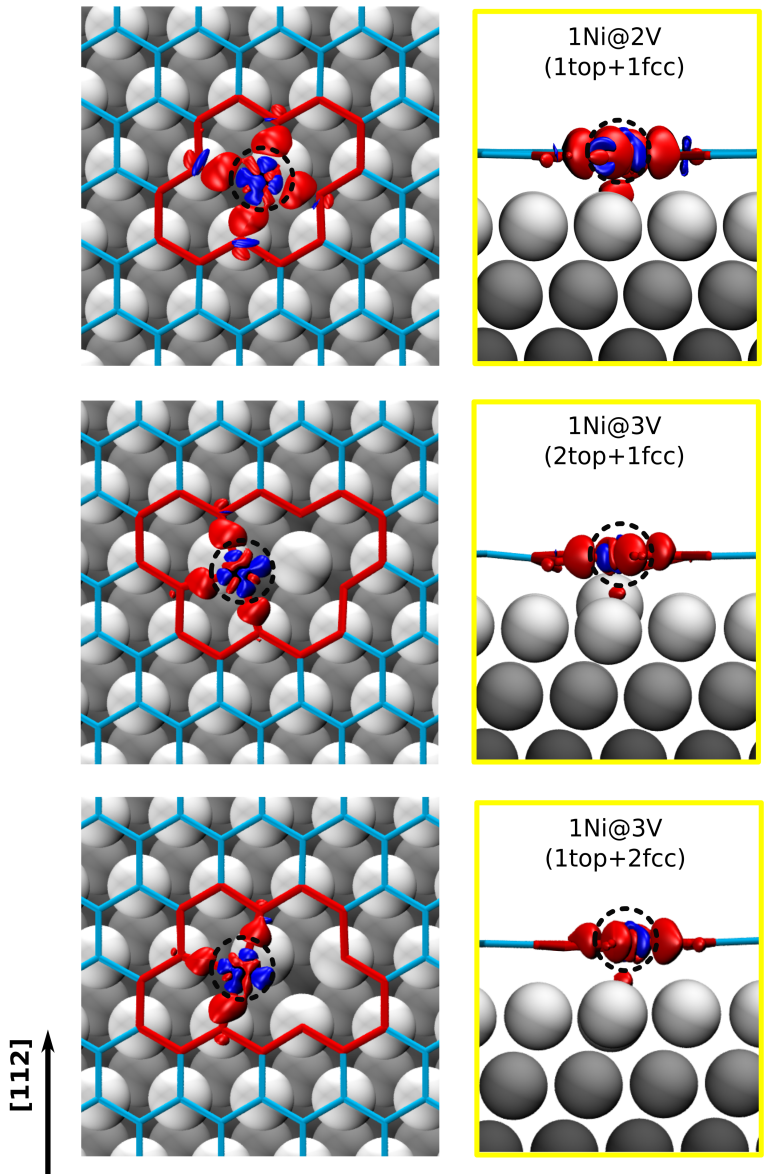

[110]

Figure 4. Electron density difference due to the filling of $2 \mathrm{~V}(1 \mathrm{top}+1 \mathrm{fcc}), 3 \mathrm{~V}(2 \mathrm{top}+1 \mathrm{fcc})$ and $3 \mathrm{~V}(1 \mathrm{top}+2 \mathrm{fcc})$ in graphene with one $\mathrm{Ni}$ adatom, obtained by subtracting to the electron density distribution of the whole defective structure the one of the structure with the empty vacancy and the one of the trapped adatom, both kept in the frozen geometry that they have in the defective structure filled by Ni. Plots of the top views (left panels) and side views along the [110] direction (right panels) are shown for 1Ni@2V(1top+1fcc), 1Ni@3V(2top+1fcc) and 1Ni@3V(1top+2fcc) structures. Red/blue indicates abundance/depletion of electrons. The electron density difference isosurfaces are plotted at $+/-0.007$ |el/a03 for 1Ni@2V(1top+1fcc), and at +/-0.01 |el/a03 for $1 \mathrm{Ni} @ 3 \mathrm{~V}(1 \mathrm{top}+2 \mathrm{fcc})$ and 1Ni@3V(2top+1fcc).

DFT calculations were performed through the Quantum ESPRESSO code, ${ }^{24}$ using plane-wave-basis set and employing the Generalized Gradient Approximation for the exchange-correlation functional in the Perdew-Burke-Ernzerhof parametrization (GGA-PBE). ${ }^{25}$ Spinpolarized calculations have been performed. In order to correctly describe the graphene/ $\mathrm{Ni}(111)$ interaction, ${ }^{26-28}$ semiempirical corrections accounting for the Van Der Waals interactions were included with the DFT-D approach. ${ }^{29}$ More refined functionals such as $\mathrm{RPBE}^{30}$ have not been considered in the present work, since previous investigations proved that the choice of the PBE functional, together with the DFT-D approach, provides an accurate description of the graphene/Ni(111) interaction.
All the calculations were performed using a periodically repeated supercell containing in plane $6 \times 6$ graphene unit cells combined with a 3-layer $\mathrm{Ni}$ slab with (111) surface: the parallel slab repeated images were separated by $15 \AA$ of vacuum. The supercell size allowed to avoid interactions between the replicas of the defects. Concerning the Brillouin zone sampling, we adopted the Methfessel-Paxton smearing technique, ${ }^{31}$ the Monkhorst-Pack kpoint mesh ${ }^{32}$ with a $2 \times 2 \times 1$ k-point grid centered on the Gamma point for the self-consistent cycles. Convergence tests suggested to adopt a kinetic energy cutoff of 30 Ry for the plane waves basis set and an energy broadening of 0.01 Ry. The theoretical equilibrium lattice parameters of graphene and $\mathrm{Ni}(111)$ surface were found to be $2.46 \AA$ and $2.49 \AA$ respectively, in excellent agreement with the experimental values. STM simulations were done using the TersoffHamann approach, ${ }^{33}$ according to which the tunneling current is proportional to the energy-Integrated Local Density of States (ILDOS). Constant-current and voltage values $\mathrm{V}_{\text {bias }}$ for the STM simulations have been chosen to match the experimental values. Stick-and-ball-model were rendered with the VMD software. ${ }^{34}$

\section{Conclusions}

In summary, we demonstrated the possibility to induce $\mathrm{Ni}$ doping into graphene during the CVD process on a Ni(111) surface. The detailed comparison between high-resolution experimental STM images and simulated images of different possible structures allowed precise identification of the atomic-scale configuration at the doping sites. Analysis of the electron density distribution shows that the $\mathrm{Ni}$ adatom is always more strongly bound to the graphene layer than to the underlying substrate, thus suggesting the possibility to maintain the doping also after decoupling from the substrate.

Our results reveal the incorporation of individual $\mathrm{Ni}$ atoms in epitaxial graphene layers during growth, showing the feasibility of doping with a transition metal by an easily scalable process, which could be applied for the doping of macroscopic graphene layers in conventional CVD plants typically used for the industrial production of graphene based devices. Moreover, we showed that in our method the growth temperature can be used as the control parameter to establish the density of doping atoms. This result paves the way to a number of new relevant applications, from spintronics to catalysis and can stimulate further studies aiming at verifying the applicability of this doping strategy to other transition metals.

\section{Conflicts of interest}

There are no conflicts to declare.

\section{Acknowledgements}

Computational resources have been obtained from CINECA through the ISCRA initiative and the agreement with the University of Trieste. We thank Giovanni Onida from the University of Milan, Italy, for fruitful discussion. 


\section{Notes and references}

1 Bae, S.; Kim, H.; Lee, Y.; Xu, X.; Park, J. S.; Zheng, Y.; Balakrishnan, J.; Lei, T.; Kim, H. R.; Song, Y. I. et al. Roll-to-roll Production of 30-Inch Graphene Films for Transparent Electrodes. Nature Nanotechnology, 2010, 5, 574-578.

2 Wei, D.; Liu, Y.; Wang, Y.; Zhang, H.; Huang, L.; Yu, G. Synthesis of n-Doped Graphene by Chemical Vapor Deposition and Its Electrical Properties. Nano Lett., 2009, 9, 1752-1758.

3 Deng, D.; Chen, X.; Yu, L.; Wu, X.; Liu, Q.; Liu, Y.; Yang, H.; Tian, H.; Hu, Y.; Du, P. et al. A Single Iron Site Confined in a Graphene Matrix for the Catalytic Oxidation of Benzene at Room Temperature. Sci. Adv., 2015, 11, e1500462.

4 Zhao, L.; Levendorf, M.; Goncher, S.; Schiros, T.; Pálová, L.; Zabet-Khosousi, A.; Rim, K. T.; Gutiérrez, C.; Nordlund, D.; Jaye, C. et al. Local Atomic and Electronic Structure of Boron Chemical Doping in Monolayer Graphene. Nano Lett., 2013 13, 4659-4665.

5 Telychko, M.; Mutombo, P.; Ondráček, M.; Hapala, P.; Bocquet, F. C.; Kolorenč, J.; Vondráček, M.; Jelínek, P.; Svec, $M$. Achieving High-Quality Single-Atom Nitrogen Ddoping of Graphene/SiC(0001) by Ion Implantation and Subsequent Thermal Stabilization. ACS Nano, 2014, 8, 7318-7324.

6 Sala, A.; Zamborlini, G.; Menteş, T. O.; Locatelli, A. Fabrication of 2D Heterojunction in Graphene via Low Energy $\mathrm{N}_{2}^{+}$Irradiation, Small, 2015, 11, 5927-5931.

7 Zhao, L.; He, R.; Rim, K. T.; Schiros, T.; Kim, K. S.; Zhou, H.; Gutiérrez, C.; Chockalingam, S. P.; Arguello, C. J.; Pálová, L. et al. Visualizing Individual Nitrogen Dopants in Monolayer Graphene. Science, 2011, 333, 999-1003.

8 Krasheninnikov, A. V.; Lehtinen, P. O.; Foster, A. S.; Nieminen, R. M., Pyykkö, P. Embedding Transition-Metal Atoms in Graphene: Structure, Bonding, and Magnetism. Phys. Rev. Lett., 2009, 102, 126807 1-4.

9 Kaukonen, M. O.; Krasheninnikov, A.; Kauppinen, E.; Nieminen, R. M. Doped Graphene as a Material for Oxygen Reduction Reaction in Hydrogen Fuel Cells: a Computational Study. ACS Catalysis, 2012, 3, 159-165.

10 Wannakao, S.; Nongnual, T.; Khongpracha, P.; Maihom, T.; Limtrakul, J. Reaction Mechanisms for CO Catalytic Oxidation by $\mathrm{N}_{2} \mathrm{O}$ on Fe-Embedded Graphene. J. Phys. Chem. C, 2012, 116, 16992-16998.

11 Guo, N.; Xi, Y.; Liu, S.; Zhang, C. Greatly Enhancing Catalytic Activity of Graphene by Doping the Underlying Metal Substrate. Sci. Rep., 2015, 5, 12058 1-7.

12 H. J. Qiu, Y. Ito, W. Cong, Y. Tan, P. Liu, A. Hirata, T. Fujita, Z. Tang, M. Chen, Nanoporous Graphene with Single-Atom Nickel Dopants: An Efficient and Stable Catalyst for Electrochemical Hydrogen Production, Angew. Chem. Int. Ed., 2015, 54, 14031-14035.

13 Wang, H.; Wang, Q.; Cheng, Y.; Li, K.; Yao, Y.; Zhang, Q.; Dong, C.; Wang, P.; Schwingenschlögl, U.; Yang, W. et al. Doping Monolayer Graphene With Single Atom Substitutions. Nano Lett., 2012, 12, 141-144.

14 Cretu, O.; Krasheninnikov, A. V.; Rodríguez-Manzo, J. A.; Sun, L.; Nieminen, R. M.; Banhart, F. Migration and Localization of Metal Atoms on Strained Graphene. Phys. Rev. Lett., 2010, 105, 196102 1-4.

15 Wan W.; Li H.; Huang, H.; Liang Wong, S.; Lv, L.; Gao, Y.; Thye Shen Wee, A. Incorporating Isolated Molybdenum (Mo) Atoms into Bilayer Epitaxial Graphene on $4 \mathrm{H}-\mathrm{SiC}(0001)$, ACS Nano, 2014, 8, 970-976.

16 Zhang, L.; Jia, Y.; Gao, G.; Yan, X.; Chen, N.; Chen, J.; Teng Soo, M.; Wood, B.; Yang, D.; Du, A.; Yao, X. Graphene Defects Trap Atomic Ni Species for Hydrogen and Oxygen Evolution Reactions. Chem, 2018, 4, 285-29.
17 Patera, L. L.; Africh, C.; Weatherup, R. S.; Blume, R.; Bhardwaj, S.; Castellarin-Cudia, C.; Knop-Gericke, A.; Schloegl, R.; Comelli, G.; Hofmann, S. et al. In Situ Observations of the Atomistic Mechanisms of Ni Catalyzed Low Temperature Graphene Growth. ACS Nano, 2013, 7, 7901-7912.

18 Africh, C.; Cepek, C.; Patera, L.L.; Zamborlini, G.; Genoni, P.; Mentes, T.O.; Sala, A.; Locatelli, A.; Comelli, G. Switchable graphene-substrate coupling through formation/dissolution of an intercalated Ni-carbide layer. Scientific Reports 2016, 6, 19734.

19 Bianchini, F.; Patera, L. L.; Peressi, M.; Africh, C.; Comelli, G. Atomic Scale Identification of Coexisting Graphene Structures on Ni(111). J. Phys. Chem. Lett., 2014, 5, 467-473.

20 Jacobson, P.; Stöger, B.; Garhofer, A.; Parkinson, G. S.; Schmid, M.; Caudillo, R.; Mittendorfer, F.; Redinger, J.; Diebold, U. Disorder and Defect Healing in Graphene on $\mathrm{Ni}(111)$. J. Phys. Chem. Lett., 2012, 3, 136-139.

21 Wang, L.; Zhang, X.; Chan, H. L. W.; Yan, F.; Ding, F. Formation and Healing of Vacancies in Graphene Chemical Vapor Deposition (CVD) Growth. J. Am. Chem. Soc., 2013, 135, 4476-4482.

22 Patera, L. L.; Bianchini, F.; Africh, C.; Dri, C.; Soldano, G.; Mariscal, M. M.; Peressi, M.; Comelli, G. Real-time Imaging of Adatom-Promoted Graphene Growth on Nickel. Science, 2018, 359, 1243-1246.

23 Patera, L. L.; Bianchini, F.; Troiano, G.; Dri, C.; Cepek, C.; Peressi, M.; Africh, C.; Comelli, G. Temperature driven changes of the graphene edge structure on $\mathrm{Ni}(111)$ : substrate vs. hydrogen passivation, Nano Lett. 2015, 15, 5662.

24 Giannozzi, P.; Baroni, S.; Bonini, N.; Calandra, M.; Car, R.; Cavazzoni, C.; Ceresoli, D.; Chiarotti, G. L.; Cococcioni, M.; Dabo, I. et al. QUANTUM ESPRESSO: a Modular and OpenSource Software Project for Quantum Simulations of Materials. J. Phys. Condens. Matter 2009, 21, 395502 1-19.

25 Perdew, J. P.; Burke, K.; Ernzerhof, M. Generalized Gradient Approximation Made Simple Phys. Rev. Lett. 1996, 77, 3865-3868.

26 Zhao, W.; Kozlov, S. M.; Höfert, O.; Gotterbarm, K.; Lorenz, M. P. A.; Viñes, F.; Papp, C.; Görling, A. Graphene on Ni(111): Coexistence of Different Surface Structures. J. Phys. Chem. Lett. 2011, 2, 759-764.

27 Kozlov, S.; Viñes, F.; Görling, A. Bonding Mechanisms of Graphene on Metal Surfaces. J. Phys. Chem. C 2012, 116, 7360-7366.

28 Cheng, D.; Barcaro, G.; Charlier, J.-C. Homogeneous Nucleation of Graphitic Nanostructures from Carbon Chains on Ni(111). J. Phys. Chem. C 2011, 115, 10537-10543.

29 Grimme, S. Density Functional Theory with London Dispersion Corrections. WIREs Comput. Mol. Sci. 2011, 1, 211-228.

30 B. Hammer, B.; Hansen, L. B.; Nørskov, J. K. Improved Adsorption Energetics within Density-Functional Theory using Revised Perdew-Burke-Ernzerhof Functionals, Phys. Rev. B 1999, 59, 7413

31 Methfessel, M.; Paxton, A. High-Precision Sampling for Brillouin-Zone Integration in Metals. Phys. Rev. B 1989, 40, 3616-3621.

32 Monkhorst, H. J.; Pack, J. D. Special Points for Brillouin-Zone Integrations. Phys. Rev. B 1976, 13, 5188-5192.

33 Tersoff, J.; Hamann, D. Theory of the Scanning Tunneling Microscope. Phys. Rev. B 1985, 31, 805-813.

34 Humphrey, W.; Dalke, A.; Schulten, K. VMD: Visual Molecular Dynamics, J. Mol. Graph. 1996, 14, 33-38. 